\title{
Use of ultra-low cost fitness trackers as clinical monitors in low resource emergency departments
}

\author{
Alon Dagan, Oren J Mechanic \\ Department of Emergency Medicine, Beth Israel Deaconess Medical Center, Harvard Medical School, \\ Boston, MA, USA
}

In low resource hospitals, strained staffing ratios and lack of telemetry can put patients at risk for clinical deterioration and unexpected cardiac arrest. While traditional telemetry systems can provide real-time continuous vital signs, they are too expensive for widespread use in these settings. At the same time, developed countries such as the United States have been increasingly utilizing remote monitoring systems to shift patient care from hospital to home. While the context is dramatically different, the challenge of monitoring patients in otherwise unmonitored settings is the same. At-home monitoring solutions range from highly comprehensive and expensive systems to inexpensive fitness trackers. In the field of global health, the adoption of this technology has been somewhat limited. We believe that low cost fitness trackers present an opportunity to address the challenge of vital sign monitoring in resource-poor settings at a fraction of the cost of existing technical solutions.

Keywords Telemedicine; Monitoring, physiologic; Fitness trackers; Internet of Things; Global health
eISSN: 2383-4625

Received: 20 September 2019 Revised: 20 September 2019

Accepted: 19 November 2019

Correspondence to: Oren J Mechanic Department of Emergency Medicine, Beth Israel Deaconess Medical Center, 1 Deaconess Rd. Rosenberg 2nd Floor, Boston, MA 02215, USA

E-mail: omechani@bidmc.harvard.edu ORCID

https://orcid.org/0000-0002-8567-0534

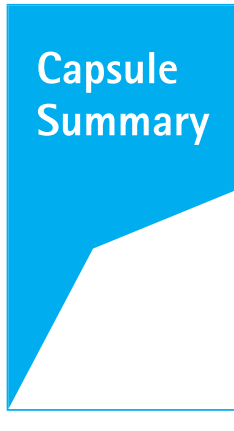

What is already known

Low-cost trackers, devices, and wearables are increasingly inexpensive and readily available. They may be excellent substitutes for high-cost telemetry monitoring in resource-poor settings.

What is new in the current study

The explosive growth in wearable health monitors presents exciting opportunities to improve care in low resource emergency department. 


\section{INTRODUCTION}

One of the many challenges facing healthcare providers in low resource settings is that of vital sign monitoring. Due in large part to overburdened hospital staff with exceptionally high nursing ratios, vital sign monitoring in developing countries often fails to occur in a reliable fashion. 'This results in many unmonitored patients at risk for clinical deterioration and adverse outcomes. In the developed world, the technology has been rapidly expanding in the area of at home and ambulatory monitoring. ${ }^{2}$ These monitoring technologies often pair with smartphones and tablets and require less infrastructure and cost than traditional hospital telemetry. With the increased ubiquity of smart devices in low- and middle-income countries, these advances have the potential to allow low resource hospitals to "leapfrog" over more traditional monitoring tools. Increasingly sophisticated monitoring solutions have been tested in these settings, but the widespread use is limited by cost and complexity. ${ }^{3,4}$ Simpler and less expensive ambulatory fitness trackers have been studied for their use in the healthcare setting, ${ }^{5,6}$ but at 150 to 250 US dollars (USD) are still too expensive for widespread use. Recently, the commercial availability of ultra-low-cost fitness trackers at prices of 20 to 40 USD presents a unique opportunity for health applications in low-resource settings. We propose an open source system utilizing commercially available ultra-low-cost heart rate monitors for clinical monitoring and outline ongoing work to test its feasibility.

\section{BACKGROUND}

Patient care in low resource clinical settings is faced with a multitude of challenges. Globally, clinical medicine is limited secondary to sparsity of medical supplies, medications, infrastructure, financial supports, and health care providers.' These limitations in infrastructure and personnel make monitoring sick patients difficult. Demands faced by nursing staff in emergency department and hospital settings in low- and middle-income countries often far exceed what would be considered safe and appropriate in developed countries. ${ }^{8,9}$ The average nurse to patient ratio in the United States and Canada is 1:4.4 on general medicine wards, and there are several states with legislation in place mandating nursing ratios of 1:1 or 1:2 for critically ill patients. ${ }^{9}$ On the other hand, studies in sub-Saharan Africa routinely demonstrate nursing ratios between 1:10 and 1:41. A study of 13 Kenyan hospitals demonstrated a mean nursing ratio of 1:31. ${ }^{8}$ With such limitations of workforce, it is often difficult to safely care for patients. In these settings, tasks as routine and fundamental as vital sign monitoring can become unattainable.
According to the Guideline for the Management of Illnesses with Limited Resources released by the World Health Organization, critically ill patients should have vital signs monitoring every 30 minutes for the first 6 hours, and once every hour after that. ${ }^{10}$ This has been found to be very difficult to accomplish in practice. In a cohort of patients with severe sepsis in Uganda, the mean number of heart rate measurements documented in 24 hours was only 1.0 (interquartile range, 0.8-1.3). ${ }^{11}$ Similarly, in a quality improvement study in two Sierra Leonean hospitals, daily vital signs were documented in only $26.6 \%$ of patients, ${ }^{1}$ and in a larger study of 13 Kenyan county hospitals more than a third (37.1\%) of pediatric admissions had no documented heart rate. ${ }^{8}$ While the magnitude of its contribution to patient outcomes is difficult to study directly, the lack of frequent vital sign monitoring certainly results in a decreased identification of clinical deterioration and disease progression. The growing science of automated and remote monitoring may offer the potential to help lift this burden from overworked nursing and hospital staff.

\section{TRADITIONAL PRACTICES IN REMOTE CARE MONITORING}

Remote care monitoring is a large segment of the United States telehealth system that aims to continuously track and record clinically significant markers of disease outside of the hospital setting. ${ }^{2}$ While the context of monitoring patients remotely in the United States is dramatically different from that of low-resource settings in developing countries, they both seek to gain limited but useful information on patients who would otherwise be unmonitored.

The remote care monitoring market has grown significantly in the United States. In conjunction with common phone applications for iOS (iPhone operating system) and Android, the industry is continuously developing bluetooth-enabled, wireless devices that allow for real-time measurement of health markers, most notably vital signs. With seamless interface with physician and hospital online medical records, these measurements can be stored to augment ongoing medical care.

Current practices in remote care monitoring aim to improve population health by identifying early markers of disease. Invasive and non-invasive home monitoring have been widely studied. These efforts include blood pressure and weights in patient with congestive heart failure, ${ }^{12,13}$ heart rate monitoring in patients with atrial fibrillation, ${ }^{14}$ and glucose readings for patients with diabetes. ${ }^{15}$ Such recordings provide meaningful informations for physicians and nurses caring for patients with ongoing medical conditions. 
With the growth of remote care monitoring in the developed world, there has been increased opportunities for its implementation in low-resource settings.

\section{USE OF MONITORING SYSTEMS IN LOW RESOURCE SETTINGS}

The increasing ubiquity of digital health monitoring in the developed world has presented an opportunity for improvement and automation in the monitoring of patients in resource poor settings. There have been several large public calls to action aimed at translating the growing capabilities of state-of-the-art health monitoring to the challenges faced in low resource settings.

In 2014, United States President Obama announced "Fighting Ebola: a grand challenge for development," launched by the US Agency for International Development in partnership with the White House Office of Science and Technology, the Centers for Disease Control and Prevention, and the Department of Defense. ${ }^{16}$ This broad initiative was aimed at addressing challenges in the diagnosis and treatment of the Ebola epidemic. One of the outcomes of this challenge was the development and implementation of a comprehensive wireless monitoring system to facilitate the care of patients in an Ebola treatment center in Makeni, Sierra Leone. ${ }^{3}$ This study was unique in its utilization of a "band-aid" patch sensor, called the MultiSense patch, developed by Rhythm Diagnostics Systems (http://www.rhythmdiagnosticsystems.com). This patch, when applied to the chest of patients, allows for the continuous monitoring of heart rate, heart rate variability, pulse oximetry, activity, respiratory rate, pulse transit time, uncalibrated skin temperature, and posture. ${ }^{3}$ This data was then wirelessly reported to healthcare providers, both in a raw form, and as an algorithm-generated Multivariate Change Index. This index, developed by physIQ (http://physiq.com) uses data driven machine learning methods to predict deviations from a patient's baseline physiology as a means of early detection of physiologic improvement or decompensation. Over a 3-week study period in the summer of 2015 , this system was able to collect 1,838 hours of continuous multiparametric waveform data. ${ }^{3}$ This study represents one of the most promising uses of a sophisticated multiparametric data collection system in a low resource setting. Unfortunately, the current system cost of 15,000 USD may limit its widespread use.

Shortly thereafter, in 2017, another highly publicized challenge, the Qualcom Tricorder XPRIZE competition was completed. This challenge was announced in 2012 and offered up to 10 million USD in prize money to develop a Star Trek style "tricorder" capable of capturing not only vital signs, but also diagnosing a subset of 12 diseases without physician input. ${ }^{4}$ The winning submission
DxtER, by Final Frontier Medical Devices (http://basilleaftech.com) successfully monitored all five vital signs as well as developed novel methods for noninvasively monitoring continuous body chemistry, and an artificial intelligence engine for diagnostics. While the award's aims were not specific to the global health context, it's potential for implementation in low resource settings is evident. Unfortunately, it has yet to be studied in such setting, and approximate costs of such a system are not publicly known.

While these cutting-edge projects are immensely promising, they remain impractical and expensive for most resource-poor settings. This is in part due to the ambitious nature of their monitoring goals. Attempting to attain comprehensive wireless monitoring as well as sophisticated diagnostics necessitates additional cost and complexity. Given the immediate needs of overworked hospital staff in low resource environments, there is perhaps a role for less sophisticated tools aimed at providing more basic functionality at a much lower cost. The rise of the inexpensive consumer fitness tracker may serve to fill this role.

\section{FITNESS TRACKERS AS VITAL SIGN MONITORS}

Wearable wrist monitors (such as Fitbit Charge, Apple Watch, Samsung Gear, and Mio Alpha) account for $87 \%$ of sold wearable devices in the United States. ${ }^{17}$ It is estimated that over 250 million smartwatches are in current use ${ }^{18}$ from over 45 manufacturers. ${ }^{5}$ These devices are non-invasive and are popularly used as fitness trackers. They utilize an inexpensive optical technique known as photoplethysmography to measure intravascular blood volume differences, and thus estimate heart rate. ${ }^{19}$ Their accuracy, cost, and utility have been heavily studied in the literature.

Several studies have been conducted aimed at comparing the monitoring capability of consumer-sold wearable devices to gold standard, electrocardiogram-leads, and accelerometers. While these studies have been quite variable, two of the largest studies have demonstrated good correlation of direct-to-consumer wearables to electrocardiogram during moderate-to-vigorous activity level (i.e., higher heart rates). ${ }^{5}$ Lowest error when compared to the gold-standard was observed for more stationary activities such as cycling (error 1.8\%, 0.9\%-2.7\%) rather than running. ${ }^{6}$

Wearable devices for activity/heart rate monitoring have also been increasingly inexpensive. Direct-to-consumer products range from 149.95 to 249.95 USD for individual sale ${ }^{20}$ and are projected to continuously decrease as competitors enter the market. While these exercise trackers are orders of magnitude less expensive than traditional medical monitoring devices, they have still been out of reach for use in very low resource areas. Recently, however, 
ultra-low-cost exercise trackers with heart rate monitoring capability have been introduced for less than 40 USD. Mi Pulse (Xiaomi, Beijing, China) is a product offered by China's largest smartphone company. ${ }^{21}$ For 19.99 USD (Amazon, 2016) Mi Band 1 and 34.99 USD (Amazon, 2018) Mi Band 2 provide an inexpensive direct-to-consumer option for wearable heart rate monitoring technology. While this extremely low price point is a tantalizing prospect for use in low-resource environments, the accuracy of these ultra-low-cost devices has yet to be studied in a clinical setting.

\section{ULTRA-LOW-COST FITNESS TRACKERS FOR CLINICAL MONITORING IN LOW-RESOURCE SETTINGS}

To the authors' knowledge, this concept of utilizing ultra-low-cost fitness trackers as heart rate monitors in clinical settings was first explored in 2016 as a part of the Health-Internet of Things Hackathon event hosted by Taipei Medical University in conjunction with Sana and the Massachusetts Institute of Technology. Please see lqbal et al. ${ }^{22}$ for details regarding this event, but in brief, this event utilized the hackathon education model to bring together clinicians and technical experts in order to address health challenges. ${ }^{22-24} \mathrm{~A}$ team lead by Dr. Kuo-Hsuan Lin, an emergency medicine attending at E-da Hospital in Kaohsiung, Taiwan, looked to tackle the challenge of unexpected cardiac arrest in his hospital system. While Taiwan is certainly not a developing country, the hospital in which Dr. Lin works is underserved and has nursing ratios reaching 1:25. In an internal unpublished quality improvement study, the hospital was found to suffer 2-4 episodes of cardiac arrest in unmonitored patients per a week. Death occurred in $59.5 \%$ of these cases. Over the course of this 2-day hackathon the team developed a prototype monitoring system utilizing ultra-low-cost Mi Pulse wrist bands. The system utilized an open source Android application running on a bluetooth enabled tablet in order to stream continuous heart rate data from several wristbands simultaneously. The team was awarded the prize for "most innovative" solution at the event and are currently looking into opportunities to implement and evaluate this concept further.

\section{PILOT STUDY}

It is with the support and permission of Dr. Lin and his hackathon team that we are currently developing a robust evaluation of the Mi Pulse wristband as an ultra-low-cost fitness tracker for use in clinical monitoring. We plan to utilize the Mi Pulse device on patients cared for in our emergency department. Consented patients undergoing telemetry monitoring as part of their routine emer- gency care will have the wristband applied, and data will be collected via Bluetooth utilizing a custom Android application. In a retrospective fashion, the accuracy of the wristband devices will be determined, both as an absolute error vs. gold standard telemetry, as well as in the identification of episodes meeting predefined alert criteria. We believe this study is unique in its assessment of an ultra-low-cost fitness monitor in a clinical setting. If successful, it presents an exciting opportunity to pair commercially available and inexpensive products with open source technology in order to dramatically increase the accessibility of vital sign monitoring in resource poor areas.

\section{DISCUSSION}

The increasing availability of ultra-low-cost fitness monitors with heart rate tracking capability presents an exciting opportunity to improve patient care in low resource settings where routine vital sign monitoring is not currently feasible. While there have been promising steps forward in complex multiparameter wireless monitors, these systems have higher costs and therefore more limited potential application. While ultra-low-cost fitness monitors will never be able to achieve the advanced comprehensive monitoring capabilities that have been demonstrated by systems such as the MultiSense patch ${ }^{3}$ or DxtER device, ${ }_{1}^{4}$ they do have the potential for an incremental improvement at a fraction of the cost. We envision the use of freely downloadable open source software that will allow providers in resource poor settings to quickly implement inexpensive off-the-shelf equipment at the bedside. We hypothesize that these devices can act as a class of "good enough" sensors that may not achieve the level of accuracy we have come to expect from medical telemetry, but can still provide clinically important and actionable information regarding changes in patient status. Through development of open source software and careful study of these devices, we hope to shed more light on these possibilities.

\section{CONCLUSION}

The explosive growth in wearable health monitors presents exciting opportunities to improve care in low resource settings. While the quest for increasing sophistication in monitoring equipment has been promising, the applicability of these systems is limited by cost and complexity. ${ }^{25}$ The commercial availability of ultra-lowcost fitness trackers presents a unique opportunity for an exceptionally simple and inexpensive intervention with broad impact. In a pilot project we plan to assess the accuracy and value of these devices to determine their utility as patient monitors in low resource settings. 


\section{CONFLICT OF INTEREST}

No potential conflict of interest relevant to this article was reported.

\section{ACKNOWLEDGMENTS}

Special thanks to the Pulse Hackathon Team: Lin Kuo-Hsuan, Department of Emergency Medicine, E-Da Hospital, Kaohsiung, Taiwan; Lin Yeng-Chih, Department of Laboratory Medicine, Lo-Hsu Medical Foundation Lotung Poh-Ai Hospital; Wu Hua-Lun, Taipei Medical University Wan Fang Hospital Department of Medical Quality.

\section{REFERENCES}

1. Rosen MA, Chima AM, Sampson JB, et al. Engaging staff to improve quality and safety in an austere medical environment: a case-control study in two Sierra Leonean hospitals. Int J Qual Health Care 2015;27:320-7.

2. Mechanic OJ, Persaud Y, Kimball AB. Telehealth systems [Internet]. Treasure Island, FL: StatPearls Publishing; 2019 [cited 2019 Apr 5]. Available from: http://www.ncbi.nlm.nih.gov/books/ NBK459384/.

3. Steinhubl SR, Feye D, Levine AC, Conkright C, Wegerich SW, Conkright G. Validation of a portable, deployable system for continuous vital sign monitoring using a multiparametric wearable sensor and personalised analytics in an Ebola treatment centre. BMJ Glob Health 2016;1:e000070.

4. Gronowski AM, Haymond S, Harris B, Peng CK, Kohli SS. A Q\&A with the Qualcomm Tricorder XPRIZE Winners. Clin Chem 2018; 64:631-5.

5. Shcherbina A, Mattsson CM, Waggott $D$, et al. Accuracy in wrist-worn, sensor-based measurements of heart rate and energy expenditure in a diverse cohort. J Pers Med 2017;7:3.

6. Ferguson T, Rowlands AV, Olds T, Maher $C$. The validity of consumer-level, activity monitors in healthy adults worn in freeliving conditions: a cross-sectional study. Int J Behav Nutr Phys Act 2015;12:42.

7. Rowe AK, de Savigny D, Lanata CF, Victora CG. How can we achieve and maintain high-quality performance of health workers in low-resource settings? Lancet 2005;366:1026-35.

8. Ogero $M$, Ayieko $P$, Makone $B$, et al. An observational study of monitoring of vital signs in children admitted to Kenyan hospitals: an insight into the quality of nursing care? J Glob Health 2018;8:010409.

9. Kane RL, Shamliyan T, Mueller C, Duval S, Wilt TJ. Nurse staff- ing and quality of patient care. Evid Rep Technol Assess (Full Rep) 2007;(151):1-115.

10. World Health Organization. IMAl district clinician manual: hospital care for adolescents and adults. Guidelines for the management of illnesses with limited resources. Geneva: World Health Organization; 2011.

11. Asiimwe SB, Okello $S$, Moore CC. Frequency of vital signs monitoring and its association with mortality among adults with severe sepsis admitted to a general medical ward in Uganda. PLoS One 2014;9:e89879.

12. Abraham WT, Stevenson LW, Bourge RC, et al. Sustained efficacy of pulmonary artery pressure to guide adjustment of chronic heart failure therapy: complete follow-up results from the CHAMPION randomised trial. Lancet 2016;387:453-61.

13. Ong MK, Romano PS, Edgington $S$, et al. Effectiveness of remote patient monitoring after discharge of hospitalized patients with heart failure: the better effectiveness after transition: heart failure (BEAT-HF) randomized clinical trial. JAMA Intern Med 2016;176:310-8.

14. Healey JS, Lopes RD, Connolly SJ. The detection and treatment of subclinical atrial fibrillation: evaluating the IMPACT of a comprehensive strategy based on remote arrhythmia monitoring. Eur Heart J 2015;36:1640-2.

15. Sacks DA, Ling Grant D, Macias M, Li X, Lawrence JM. The virtual office visit for women with gestational diabetes mellitus. Diabetes Care 2017;40:e34-5.

16. Fast L, Waugaman A. Fighting Ebola with information: learning from data and information flows in the West Africa Ebola response [Internet]. Washington, DC: US Agency for International Development; 2016 [cited 2019 Jan 1]. Available from: https://www.usaid.gov/sites/default/files/documents/15396/ FightingEbolaWithlnformation.pdf.

17. Wallen MP, Gomersall SR, Keating SE, Wisloff U, Coombes JS. Accuracy of heart rate watches: implications for weight management. PLoS One 2016;11:e0154420.

18. Smartwatches and smart bands dominate fast-growing wearables market [Internet]. [place unknown]: CCS Insight; 2014 [cited 2019 Feb 18]. Available from: https://www.ccsinsight. com/press/company-news/1944-smartwatches-and-smartbands-dominate-fast-growing-wearables-market/.

19. Allen J. Photoplethysmography and its application in clinical physiological measurement. Physiol Meas 2007;28:R1-39.

20. Evenson KR, Goto MM, Furberg RD. Systematic review of the validity and reliability of consumer-wearable activity trackers. Int J Behav Nutr Phys Act 2015;12:159.

21. The China smartphone market picks up slightly in 201404 IDC Reports [Internet]. Surrey: Display Daily; 2015 [cited 2019 
Feb 18]. Available from: https://www.displaydaily.com/article/ press-releases/the-china-smartphone-market-picks-up-slightly-in-2014q4-idc-reports.

22. lqbal U, Dagan A, Syed-Abdul S, et al. A hackathon promoting Taiwanese health-IoT innovation. Comput Methods Programs Biomed 2018;163:29-32.

23. Angelidis $P$, Berman $L$, Casas-Perez $M L$, et al. The hackathon model to spur innovation around global mHealth. J Med Eng Technol 2016;40:392-9.
24. Lyndon MP, Cassidy MP, Celi LA, et al. Hacking hackathons: preparing the next generation for the multidisciplinary world of healthcare technology. Int J Med Inform 2018;112:1-5.

25. Mechanic OJ, Kurtzman ND, Chiu DT, Nathanson LA, Berkowitz SJ. Point of care image capture with a custom smartphone application: experience with an encounter-based workflow. J Digit Imaging 2020;33:83-7. 